\title{
Correction to: Novel synthesis method for photo-catalytic system based on some 3d-metal titanates
}

\author{
Yahya Absalan' ${ }^{1}$ Irena G. Bratchikova ${ }^{2}$ - Nikolai N. Lobanov ${ }^{3}$. \\ Olga V. Kovalchukova ${ }^{1}$
}

Published online: 7 October 2017

(C) Springer Science+Business Media, LLC 2017

\section{Correction to: J Mater Sci: Mater Electron}

\section{DOI 10.1007/s10854-017-7769-6}

The original version of this article unfortunately contained an error in one of the co-author's name. The third author's name should read as "Nikolai N. Lobanov" instead of "Nikolay N. Lobanovc".

The original article was corrected.

The online version of the original article can be found under doi:10.1007/s10854-017-7769-6.

Yahya Absalan

yahyaabsalan2014@gmail.com

1 General Chemistry Department, RUDN University, 6 Miklukho-Maklaya st., Moscow, Russia 117198

2 Physical and Colloidal Chemistry Department, RUDN University, 6 Miklukho-Maklaya st., Moscow, Russia 117198

3 Inorganic Chemistry Department, RUDN University, 6 Miklukho-Maklaya st., Moscow, Russia 117198 